\title{
NOTE
}

\section{CALCULATION OF THE ABSORPTION COEFFICIENT FOR LINES WITH COMBINED DOPPLER AND LORENTZ BROADENING}

\author{
Charles Young
}

High Altitude Engineering Laboratory, Department of Aeronautical and Astronautical Engineering, University of Michigan, Ann Arbor, Michigan, U.S.A.

(Received 25 November 1964)

Abstract-Methods are described for calculating the spectral absorption coefficient for lines with combined Doppler and Lorentz broadening.

RADIATIVE transfer calculations involving gases at low pressures require values for the absorption coefficient incorporating Doppler and collisional broadening. The appropriate absorption coefficient can be written (cf. PENNER ${ }^{(1)}$ ),

$$
k_{v}=\frac{k_{0} y}{\pi} \int_{-\infty}^{\infty} \frac{\exp \left[-t^{2}\right]}{y^{2}+(x-t)^{2}} d t
$$

where

$$
\begin{aligned}
& k_{0}=\frac{S}{\alpha_{D}}\left(\frac{\ln 2}{\pi}\right)^{1 / 2} \\
& y=\frac{\alpha_{L}}{\alpha_{D}}(\ln 2)^{1 / 2} \\
& x=\frac{\nu-\nu_{0}}{\alpha_{\nu}}(\ln 2)^{1 / 2} \\
& S=\text { the line strength } \\
& \alpha_{D}=\frac{\nu_{0}}{c}\left(\frac{2 k T \ln 2}{m}\right)^{1 / 2}, \text { the Doppler half-width } \\
& \alpha_{L}=\text { Lorentz half-width } \\
& \nu_{0}=\text { wave number of the line center } \\
& \nu=\text { wave number at which } k_{v} \text { is to be evaluated. }
\end{aligned}
$$

Unfortunately (1) is not easy to integrate for the range of $x$ and $y$ which would apply to a planetary almosphere. PtNNEk ${ }^{(1)}$ discusses and references some methods for evaluating (1) which are rather restricted in their range of applicability and accuracy. Some recent tabulations are given by Posener, ${ }^{(2)}$ Faddeeva and Terentev ${ }^{(3)}$ and Fried and 
CONTE. ${ }^{(4)}$ Tables are not particularly convenient if radiative transfer calculations are being performed on a digital computer. SHVED and TSARITSYNA ${ }^{(5)}$ detail a procedure for calculating (1); their method, however, appears inefficient for routine use on a digital computer. The method used by FADDEEVA and TERENTEV ${ }^{(3)}$ to generate their tables is not readily adaptable for computer use. FRIED and CONTE, ${ }^{(4)}$ on the other hand, generated their tables using a digital computer and a rather elegant method. A variation of their method was used to evaluate (1) over part of the range of interest.

The integral appearing in (1) is related to the probability integral for complex argument,

$$
w(z)=\frac{1}{1 \pi} \int_{0}^{x} \frac{\exp \left[-t^{2}\right]}{t-z} \mathrm{~d} t
$$

where

$$
z=(x+i y): u(z)=u(x, y)+i x(x, y)
$$

It is easy to show (cf. FaddeEva and Terentr. ${ }^{(3)}$ ) that

$$
\frac{k_{v}}{k_{0}}=\frac{r(x, r)}{1 \pi}
$$

Equation (2) may also be expressed as a differential equation

$$
w^{\prime}(z)+2 z w(z)+2 i=0
$$

with the initial condition $\mu(0)=i, \pi$. Equation (3) may be alternatively written in the form

with initial conditions

$$
\begin{aligned}
& \frac{u}{x}+2 x u-2 u+2=0 \\
& \frac{x}{i x}+2 x u+2 y u=0
\end{aligned}
$$

$$
u(0,0)=0, \quad v(0.0)=1 \pi
$$

Integrating (4) along the $y$-axis allows $u(0, y)$ and $r(0, y)$ to be determined while integrating along the $x$-axis gives $u(x, 0)$ and $v(x, 0)$. The values obtained are

and

$$
u(0, y)=0, \quad u(0, y)=\imath(\pi) \exp \left[y^{2}\right](1-\operatorname{erf}(y))
$$

$$
u(x, 0)=-2 \exp \left[-x^{2}\right] \int_{0}^{x} \exp \left[t^{2}\right] \mathrm{d} t, \iota(x, 0)=\nu(\pi) \exp \left[-x^{2}\right]
$$

To evaluate (2) for $y \leqslant 1$, FRIED and CONTE ${ }^{(4)}$ solved the differential equations (4) using a numerical technique. Knowing $u(0, y)$ and $v(0, y)$ they integrated in the $x$-direction until the desired value of $x$ was reached. The integrating step size was $0 \cdot 01$. This method is satisfactory for producing a set of tables but on a routine basis it would involve a large amount of computer time for even moderately large values of $x$. Also round-off error is increased if a large number of integrating steps are used. The method adopted was to integrate in the $y$-direction using $u(x, 0)$ and $v(x, 0)$ as initial values. Since $y$ will not be 
greater than unity reasonably few iterations are required. It was determined that a step size of 0.02 gave six significant figure accuracy if a fourth order Runge-Kutta procedure was used. The value $v(x, 0)$ is readily calculated but $u(x, 0)$ is somewhat more difficult. For valucs of $x \geqslant 4.5$ an asymptotic cxpansion (ERDELYI et al. ${ }^{(6)}$ ) was used,

$$
\exp \left[-x^{2}\right] \int_{0}^{x} \exp \left[t^{2}\right] \mathrm{d} t=\frac{1}{2}\left[\sum_{m=0}^{M}-\frac{\left(\frac{1}{2}\right)_{m}}{x^{2 m+1}}\right]
$$

where

$$
\begin{aligned}
& (a)_{n}=a(a+1) \ldots(a+n-1), n=1,2,3, \ldots \\
& (a)_{0}=1
\end{aligned}
$$

The number of terms needed to obtain seven significant figure accuracy is given approximately by $2+40 / x$. For $2 \cdot 0<x<4.5$ an expansion in terms of Chebyshev polynomials given by HUMmer ${ }^{(7)}$ was used. This expansion is very accurate, fourteen significant figure accuracy being obtained if all the terms are included. Seven significant figure accuracy being obtained by taking the first 21 terms. For values of $x \leqslant 2 \cdot 0$ a convergent series expansion (ERDÉLYI et al. ${ }^{(6)}$ ) was used,

$$
\exp \left[-x^{2}\right] \int_{0}^{x} \exp \left[t^{2}\right] \mathrm{d} t=\sum_{n=0}^{\infty} \frac{(-1)^{n} x^{2 n+1}}{\left(\frac{3}{2}\right)_{n}}
$$

The number of terms required for seven significant figure accuracy is approximately $\left(12+5 x^{2}\right)$. Both of the above series were evaluated by nesting the sums to reduce roundoff error.

FRIED and CONTE ${ }^{(4)}$ give a continued fraction expansion for (2), derived using the quotient difference algorithm, applicable for $y>0$. It can be written

where

$$
w(z)=\lim _{n \rightarrow \infty} \frac{A_{n}}{B_{n}}
$$

$$
\begin{gathered}
A_{n+1}=b_{n+1} A_{n}+a_{n+1} A_{n-1} \\
B_{n+1}=b_{n+1} B_{n}+a_{n+1} B_{n-1} \\
A_{-1}=1: A_{0}=0: B_{-1}=0: B_{0}=1 \\
a_{n+1}=-n(2 n-1) / 2, \quad n=1,2, \ldots \\
a_{1}=z \\
h_{n+1}=-z^{2}+\frac{1}{2}+2 n, n=0,1,2, \ldots
\end{gathered}
$$

The continued fraction expansion converges slowly for $y \leqslant 1$ but rapid convergence is obtained for $y>1$. For example for $y=10 \cdot 0, x=10 \cdot 0$ only three terms are requircd for six significant figure accuracy and only six terms are required for the same accuracy for $y=2 \cdot 0, x=0 \cdot 5$. The continued fraction expansion was used for $y>1 \cdot 0$. 
It was determined that the Runge-Kutta method became unstable for $x>50 \cdot 0$. However, equation (1) is in a form suitable for integration using Hermite-Gauss quadrature (cf. HildeBRAND ${ }^{(8)}$ ). Unfortunately Hermite-Gauss quadrature is satisfactory only for fairly large $x$ or $y$ due to the very sharp peak in the curve exp $\left[-t^{2}\right] /\left(y^{2}-(x-t)^{2}\right)$ vs. $f$ for small $x$ and $y$. It was found that for $x>10 \cdot 0, y \geqslant 0,20$-point Hermite-Gauss quadrature gave results comparable to the Runge-Kutta and continued fraction expansion. The disagreement varied from 2 to 5 in the sixth significant figure except for small values of $y(y<0 \cdot 01)$ and large $x(x>20)$ when the disagreement became larger, up to 3 in the fifth significant figure. In these cases $k_{v} k_{0}$ was in the range $10^{-5}$ to $10^{-7}$ so this error was considered negligible.

The results obtained were compared with the values given by FADDEEVA and TIRINTIV ${ }^{(3)}$ and FrIFD and CONTE. ${ }^{(4)}$ Complete agreement was obtained. Needless to saly when the above procedure was being developed the three methods used to evaluate (1) were checked by overlapping the regions they covered, excellent agrecment was obtained.

To sum up, equation (1) was evaluated for

$y \leqslant 1 \cdot 0, x<10 \cdot 0$ using Runge-Kutta method

$y>1 \cdot 0, x<10 \cdot 0$ using continued fraction expansion

$y \geqslant 0, x \geqslant 10 \cdot 0$, using 20 -point Hermite-Gauss quadrature.

A Fortran program and tabulations of the integral in (1) for selected values of $x$ and $y$ can be obtained by contacting the author.

Acknowledgements. This work was supported by NASA under contract NASr-54(03). The author is grateful to $S$. ROLAND DRAYSON and PAUL B. HAYs for several interesting discussions and to Davin 1 . CHI Ds for translating the MAD* program into an efficient assembly language (UMAP $\nrightarrow$ ) program.

\section{REFERENCES}

1. S. S. PENNer, Quantitative Moleculat Spectroscopy and Gas Emissivities. Addison-Wesley, Reading. Mass (1959).

2. D. W. Posfener, Aust. .I. Phys. 12, 184 (1959).

3. V. N. Fndderva and N. M. Terentey, Tables of the Probability Integral for Complex Aigmment. Pergamon Press, New York (1961).

4. B. D. FrJed and S. D. Conte, The Plasma Dispersion Function. Academic Press, New York (1961).

5. G. M. Shved and I. V. Tsaritsyna, Problems of the Physics of the Atmosphere, Collection I. Leningrad University, pp. 106 169. NASA Technical Translation TT F-184 (1963).

6. A. Erdflyi, W. Magnus, F. Oberhettivger and F. G. Tricomi, Higher Transcendental Functions, Vol. 2, p. 147. McGraw-Hill, New York (1953).

7. D. G. Hummre, Math. Comp. 18, 317 (1964).

8. F. B. Hildebrand, Introduction to Numerical Andysis. McGraw-Hill. New York (1956).

* MAD-Michigan Alogorithm Decoder, a programming language sinilar to Fortran.

+ UMAP University of Michigan Assembly Program, an assembly language similar to FAP. 